The necessity thus arises for a more detailed study of the mechanism of transition of kinetic into vibrational energy. It seems to us to be interesting (1) to study directly the distribution of speeds of the ions once reflected from monoatomic and diatomic molecules; (2) to study the critical potentials of the radiation of polar molecules excited by collisions with ions, and (3) to investigate the thermal capacity of a stream of diatomic gas, preserved from contact with the walls by a concentric stream of monoatomic gas.

We intend to carry out investigations in these directions. The question as to transition of kinetic into vibrational energy is a fundamental one in chemical kinetics. Therefore we intend also to determine the energy of ions at which begins the decay of some molecules, the kinetics of which follows the monomolecular law, as well as to determine at which energy of the ions some reactions begin which require a previous dissociation of one of the molecules.

\section{N. SemenofF.}

A. SHECHTER.

State Phys.-Tech. Institut,

Leningrad, July 14.

A. Leipunsky u. A. Shechter, Zeit. f. Phys., 59, 857; 1930.

\section{Foaming of Beer.}

Some little time ago, seeking refreshment in a wayside tavern, I heard a man give an order in a most implacable manner, for a "half pint of bitter and in a dry glass if you don't mind ". With great zest and concentrated attention this connoisseur drank his ale and departed, leaving me free to question the landlady on the merits of a dry glass. She explained that some people asked for a dry glass because they liked a head on their beer, and on occasion when Bass's, Guinness's, and the like were a bit fiery, they were compelled to wet the glasses to keep them down.

I have verified this fact by questions of other ale drinkers and I have discussed the matter with three physicists of standing to whom the matter was novel.

The froth on beer normally assumes a peaked cap, showing what might be the effects of a viscous drag on the sides of the glass, but the effect may not be due to viscosity so much as wetting action with film tensions against the glass walls. The matter is possibly worthy of investigation in connexion with industrial processes where foaming liquids are used, and more generally, in connexion with ebullition, the wetting of the walls by a liquid different from that contained in the vessel may be interesting or useful.

I confess that I write this with mischievous but respectful curiosity. Is there more than one very important journal to which such a contribution could be addressed ?

39 Spencer Road,

Chiswick, W.4.

THE foaming or frothing of beer has been discussed fairly extensively from time to time, although the point now raised by Dr. Rowell does not appear to have been specifically mentioned. The actual frothing is, no doubt, due to a lowering of the surface tension of the beer, but the primary cause of this lowering has not been definitely established. It is most likely due to the concentration of certain dissolved constituents, such as peptones and albumoses, being greater in the surface layers of the beer than in the bulk of the beer. The retention of the dissolved carbon dioxide in the froth may also assist in the formation of the 'head', although this view is not accepted by some observers. Very small traces of impurities, such as oil, grease, or higher alcohols, disturb surface tension, and the wetted surface of a glass may act similarly.

At the Government Laboratory we have carried out a few experiments with a number of ales and stouts, and the results agree with the facts stated in the early portion of Dr. Rowell's letter, although in some instances the difference between the size of the 'head ' in the 'wet' and 'dry' glasses was not very marked.

R. RobERTSON.

\section{Infection of Phlebotomus perniciosus Newstead with Leishmania infantum.}

Although a considerable amount of research has been carried out in recent years on the transmission of kala-azar in China and India, very little has been added to the problem of the transmission of infantile kala-azar in the Mediterranean basin, apart from the infection of Phlebotomus papatasii, $P$. perniciosus var., and $P$. major on cultures of Leishmania infantum.

Working in Catania in Prof. Longo's clinic, we were able to infect $P$. papatasii and $P$. perniciosus (wild and laboratory bred) on a Chinese hamster infected with local strains of $L$. infantum during the earliest stages of the infection.

Out of 18 ? $P$.perniciosus (5 wild and 13 laboratory bred), 15, that is, 83 per cent, became infected. Control, 319 오 wild $P$. perniciosus were dissected and found negative. Out of 123 o $P$. papatasii (51 wild and 72 laboratory bred) fed on the same hamster, only one became infected. Control, 26949 . wild $P$. papatasii were dissected and found negative.

In both $P$. perniciosus and $P$. papatasii the flagellates tended to adopt an anterior position; in $P$. perniciosus at a temperature of $29^{\circ} \mathrm{C}$. to $30^{\circ} \mathrm{C}$. flagellates were found in the pharynx three and a half days after the infecting feed.

Wild $P$. papatasii was also infected by feeding through membranes on infected human bone marrow. It was found that there was occasionally a slight infection rate if smears of the bone marrow showed an average of at least one Leishman-Donovan (L.D.) body per twenty fields (Oc. $4 \mathrm{Obj} \cdot \frac{1}{12}$ ) and no infection with one L.D. body per forty fields. By culture methods we could demonstrate the presence of L.D. bodies in the circulating blood in almost 100 per cent of cases of infantile kala-azar (in some cases with only 5 c.mm. of blood), but a concentration as heavy as one L.D. body per forty fields in the blood has never yet been observed by anybody in this disease. $P$. papatasii can therefore be excluded as an important vector of $L$. infantum. Up to the present we have not succeeded in producing an infection rate of more than 26 per cent in wild $P$. papatasii after feeding on heavily infected bone marrow.

$P$. perniciosus takes on an average a much smaller feed than $P$. papatasii, and the high infection rate produced in $\mathcal{P}$. perniciosus by relatively few L.D. bodies shows that $L$. infantum is particularly well adapted to this sandfly, which should be considered as a good carrier of kala-azar in Italy.

\section{S. AdLer.}

O. THEODOR.

Kala-Azar Commission of the Royal Society and Hebrew University of Jerusalem.

\section{The Quantum and Vision.}

THe statement is frequently made that one quantum of light delivered instantaneously is just sufficient to excite the sensation of vision. We have made some measurements on the subject, and find that this is not the case.

By working with a pinhole aperture in a light-tight room, we found that a steady source equivalent to

No. 3177 , VoL. 126] 